\title{
Self-Healing Hydrogels Based on Carboxymethyl Chitosan and Acryloyl-6-aminocaproic Acid
}

\author{
Jiufang Duan \\ Institute of Material Science and Technology, Beijing Forestry University, Beijing 100083, China \\ Correspondence should be addressed to Jiufang Duan; duanjiu99@163.com
}

Received 13 May 2015; Accepted 28 July 2015

Academic Editor: Pasquale Longo

Copyright ( 2015 Jiufang Duan. This is an open access article distributed under the Creative Commons Attribution License, which permits unrestricted use, distribution, and reproduction in any medium, provided the original work is properly cited.

\begin{abstract}
Once cracks have formed within hydrogel materials, the integrity of the structure is signifcantly compromised, regardless of the application. Here, we demonstrate cross-linked CMCS hydrogels can be engineered to exhibit self-healing under mild conditions. CMCS hydrogels based on CMCS and acryloyl-6-aminocaproic acid (A6ACA) were synthesized by free radical aqueous copolymerization using ammonium persulfate as initiator. A series of hydrogels was synthesized varying the percentage of A6ACA. The hydrogels were characterized by Fourier transform infrared spectroscopy (FTIR) techniques and their morphologies were investigated by scanning electron microscope (SEM) images. When the proportion of A6ACA was increased, the compressive strength, stress, and strain of hydrogels were increased. The cross-linked hydrogel based on CMCS that can autonomously heal between cut surfaces after $1 \mathrm{~h}$ was formed under mild conditions. The increase of A6ACA content in the hydrogels will lead to increased mechanical properties and mechanical healing efficiencies for highly cross-linked polymeric networks. Hydrogen bond is the main reason for self-healing ability, and the covalent cross-linkss and noncovalent cross-links both bear loads in the hyrogel. Polymers with the ability to self-repair after sustaining damage could extend the lifetime of materials used in many applications.
\end{abstract}

\section{Introduction}

Hydrogels consist of three-dimensional hydrophilic polymer networks in which a large amount of water is interposed. Because of their unique properties, a wide range of medical, pharmaceutical, and prosthetic applications has been proposed for them [1-3]. Carboxymethyl chitosan (CMCS) has many unique chemical, physical, and biological properties such as low toxicity, biocompatibility, and good ability to form films, fibers, and hydrogels [1-3]. Hydrogels with the ability to repair themselves after sustaining damage could extend the lifetime of materials used in many applications. So far, selfhealing has been demonstrated in linear polymers, host-guest polymers $[4,5]$, supramolecular networks $[6,7]$, dendrimerclay systems [8], metal ion-polymer systems [9], and multicomponent systems [10-12]. In recent years there has been intense research into supramolecular network induced selfhealing materials [13-15].

Supramolecular network is an effective way to prepare self-healing materials. However, the self-healing CMCS hydrogels based on cross-linked network integrated by physical or chemical methods have seldom been reported. It would be interesting to study the preparation of self-healing CMCS hydrogels by an easy method. Here we describe a strategy for introducing physical and chemical cross-links into a synthetic CMCS network and demonstrate that such a network indeed displays self-healing properties.

\section{Experimental}

2.1. Material and Methods. Carboxymethyl chitosan, aminocaproic acid, acryloyl chloride, and acrylamide, ammonium persulfate, and $\mathrm{N}, \mathrm{N}^{\prime}$-methylenebisacrylamide were of analytical grade and used without further purification.

IR spectra were recorded by FTIR (Nicolet iN10 Thermo Fisher Scientific, China) in the region of $400-4000 \mathrm{~cm}^{-1}$. The morphological characterization of gel was performed with scanning electron microscopy (S-3400 N, HIACHI, Japan); the gravimetric method was employed to measure the swelling ratios of the gels in distilled water at $25^{\circ} \mathrm{C}$. After 


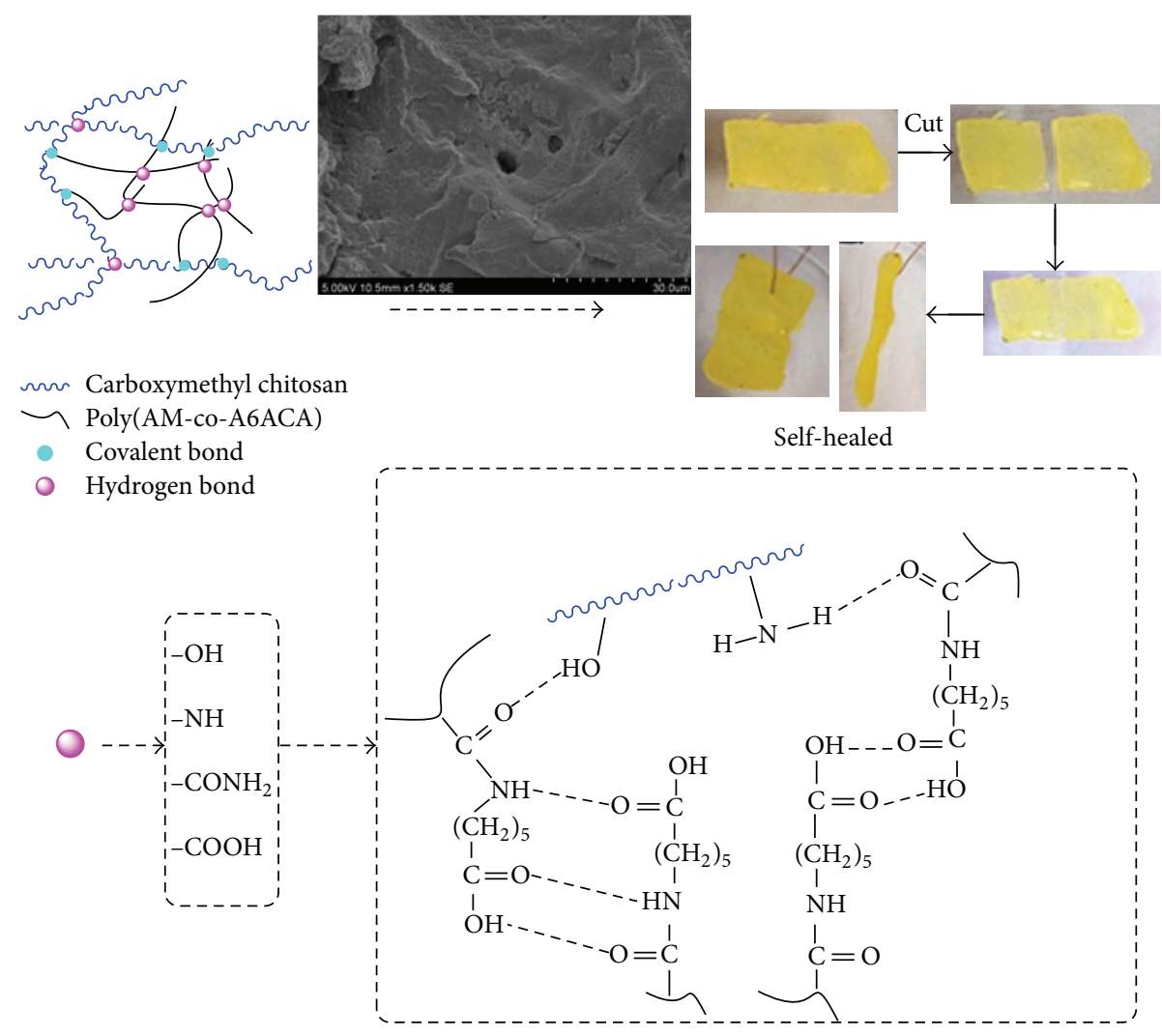

FIGURE 1: Schematic illustration of the network in hydrogels.

immersion in distilled water for about $48 \mathrm{hr}$ to reach swelling equilibrium, the gel samples were taken out and weighed after removing the excess water on the surfaces. Each data was measured in three samples, and the average value of three measurements was taken. The equilibrium swelling ratio (SR) was calculated as $\mathrm{SR}=W_{s} / W_{d}$, where $W_{s}$ is the weight of the swollen gel and $W_{d}$ is the weight of the gel at the dry state; mechanical property was measured using an Instron 3365 Universal Testing Machine (Norwood, MA) with the following parameters: sampling rate, $10.000 \mathrm{pts} / \mathrm{sec}$; beam speed, $1.0000 \mathrm{~mm} / \mathrm{min}$; full scale load range, $0.1000 \mathrm{kN}$; humidity, $25 \%$; and temperature, $23^{\circ} \mathrm{C}$.

\subsection{Preparation of Hydrogel}

2.2.1. Synthesis of A6ACA. $0.1 \mathrm{~mol} 6$-aminocaproic acid and $0.11 \mathrm{~mol} \mathrm{NaOH}$ were dissolved in $80 \mathrm{~mL}$ deionized water in ice bath under vigorous stirring. To this, $0.11 \mathrm{~mol}$ acryloyl chloride in $15 \mathrm{~mL}$ tetrahydrofuran was added dropwise. The $\mathrm{pH}$ was maintained at 7.5 until the reaction was complete. The reaction mixture was then extracted with ethyl acetate. The clear aqueous layer was acidified to $\mathrm{pH} 3.0$ and then extracted again with ethyl acetate. The organic layers were collected, combined, and dried over sodium sulfate. The solution was then filtered, concentrated, and precipitated in petroleum ether. Further purification was achieved by repeated precipitation and the product was lyophilized [16].
2.2.2. Synthesis of Hydrogels. Hydrogels were prepared by free radical polymerization in aqueous solution containing certain amounts of $86 \mathrm{wt} . \%$ distilled water, reactants (CMCS : A6ACA = 1:1 mass ratio), 0.6 wt.\% $\mathrm{N}, \mathrm{N}^{\prime}$-methylenebisacrylamide, and $0.5 \mathrm{wt} . \%$ ammonium persulfate that were added to a three-neck reactor and dissolved in solution, polymerized for $24 \mathrm{~h}$ at $37^{\circ} \mathrm{C}$.

\section{Results and Discussion}

3.1. Preparation and Structure of Hydrogels. During the free radical polymerization, the reactive double bonding groups on the surface of CMCS form highly branched polymer networks, which lead to CMCSs acting as multifunctional crosslinks and bridge the adjacent CMCSs. As a result, the intertwining of polymeric chains was promoted and the hydrogenbonding interaction between hydrophilic groups such as $-\mathrm{COOH},-\mathrm{CONH}_{2},-\mathrm{NH}_{2}$, and $-\mathrm{OH}$, among others, was strengthened (Figure 1). Thus, the degree of cross-linking was increased, fact that is extremely favorable to the improvement of mechanical properties. The cross-linked hydrogel based on CMCS that can autonomously heal between cut surfaces after $1 \mathrm{~h}$ was formed under mild conditions (Figure 1). However, A6ACA was main reason for the efficient and robust healing of hydrogels, the side chain was sufficiently long and flexible, and the network is sufficiently deformable, to make the 


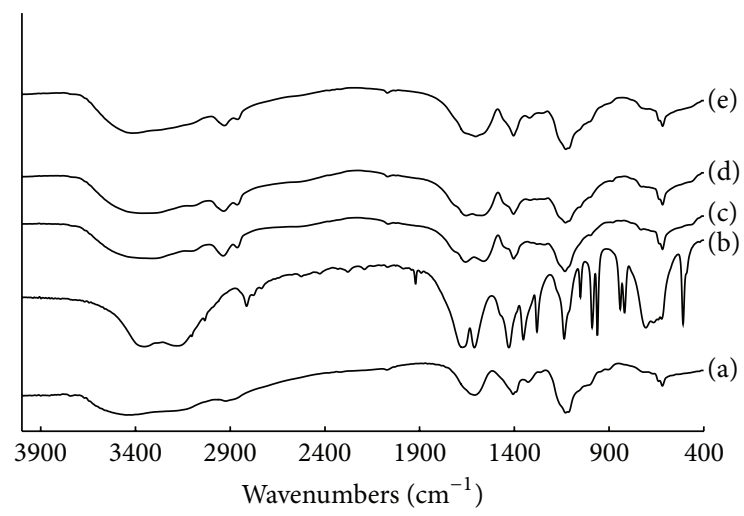

FIGURE 2: FTIR spectra of hydrogels with different A6ACA content. (a) CMCS; (b) A6ACA; (c) 90\%wt.\%; (d) 80\% wt.\%; (e) 50\% wt.\%.

functional groups across the interface accessible to each other beyond the corrugation of the interface [15].

Figure 2 shows the FTIR spectra of the unmodified chitin and a series of products obtained by the grafting of CMCS with A6ACA and AM. As shown in Figure 2, the peaks for CMCS are at $1654 \mathrm{~cm}^{-1}$ for the $\mathrm{C}=\mathrm{O}$ bonds of the carboxyl groups. After free radical copolymerization with A6ACA, the spectra showed several new absorption bands in addition to the original peaks of pure CMCS (Figures 2(c), 2(d), and 2(e)). The new absorption band at $1715 \mathrm{~cm}^{-1}$ was assigned to the $\mathrm{C}=\mathrm{O}$ stretching vibration of the amide group, and those appearing at $1562 \mathrm{~cm}^{-1}$ and $1404 \mathrm{~cm}^{-1}$ were assigned to the asymmetric and symmetric stretching vibrations, respectively [17]. This clearly indicates that CMCS grating of polyA6ACA was successfully performed.

3.2. Mechanical Properties. All mechanical tests were performed in air, at room temperature, using a tensile machine with a $100 \mathrm{~N}$ load cell. The gel specimens were made into cube shape measuring $10 \times 10 \times 10 \mathrm{~mm}^{3}$.

We prepared hydrogels containing various proportions of water to study the best ratio of the hybrids which lead to the stretchable and strong properties. When the proportion of water was increased, the elongation at break and the strain of the hybrid hydrogel increased and then decreased from 86 wt.\% (Figure 3). However, the critical stretch at rupture reached a maximum at $86 \mathrm{wt} . \%$ water. When the water content is relatively low, the molecular chains will not effectively stretch, which result in the reactive group contact insufficiently; also when the water content is too high, monomer functional groups will not collide effectively owing to lower concentration, which result in low cross-linking density in both cases. Correspondingly, the mechanical properties are poor. Unless otherwise stated, the water content was fixed at 86 wt.\%.

One effective way to improve the mechanical properties of hydrogels was increasing the cross-linking density [18]. When the proportion of A6ACA was increased, the compressive strength, stress, and strain were increased (Figures 4(a) and 4(b)). With increasing amounts of A6ACA from $10 \mathrm{wt} . \%$

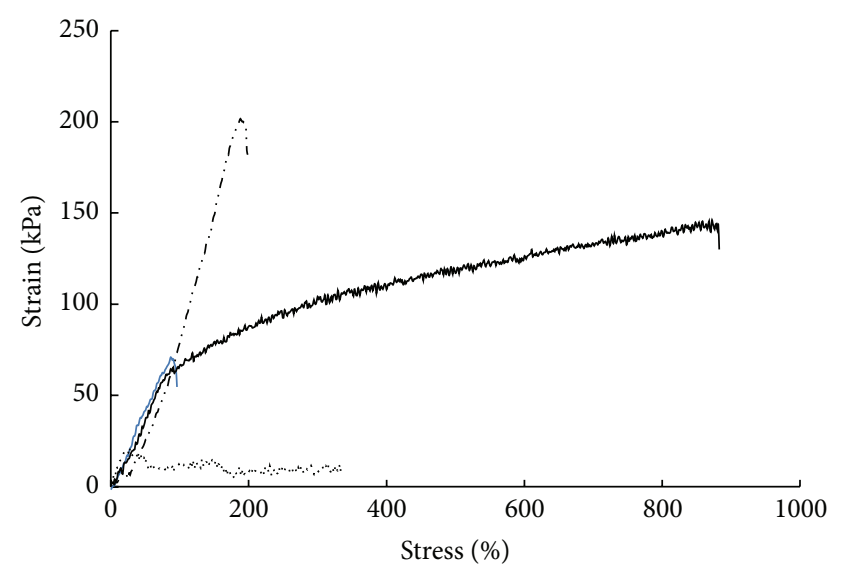

$\begin{array}{lll} & \text { (a) } & \\ \ldots \text { (b) } & \text { (c) }\end{array}$

FIGURE 3: Effects of water content on mechanical properties of hydrogels (water content: (a) 80 wt.\%; (b) 83 wt.\%; (c) 86 wt.\%; (d) 90 wt.\%).

to $80 \mathrm{wt} . \%$, the elongation at break and strain increased from $47 \%$ to $101 \%$ and $76 \mathrm{KPa}$ to $850 \mathrm{KPa}$, respectively (Figure 4(a)). However, the critical stretch at rupture reached a maximum at $80 \mathrm{wt} . \%$ A6ACA. The densities of noncovalent and covalent cross-links also strongly affect the mechanical behaviour of the hydrogels. The increased compressive strength, strain, and elongation at break were consistent with the expected increases in the cross-linking densities in the three-dimensional network structure. The hydrogen bonding in physical or chemical covalent cross-linked network was strengthened by more A6ACA, because the A6ACA provides both vinyl groups, which can form covalent bonds, and $-\mathrm{CONH}_{2},-\mathrm{NH}-$, and $-\mathrm{COOH}$ groups, which can form hydrogen bonding [15]. More A6ACA in matrix will correspondingly improve the mechanical properties of hydrogels.

3.3. Absorbency of Hydrogels. We prepared hydrogels containing various proportions of A6ACA to study the effect of A6ACA on the water absorbing capacity of hydrogels. According to Flory's theory [19], cross-linking density is a key factor influencing the ability of water absorption hydrogels. According to Figure 5, there is a maximum swelling ratio at A6ACA 30 wt.\% amount. Increasing the A6ACA amount from $10 \mathrm{wt} . \%$ to $30 \mathrm{wt} . \%$, the swelling ratio was increased because the low content of A6ACA enhanced the hydrophilicity of the hydrogel and caused a greater affinity for water, which was originated from the more hydrophilic groups generated from A6ACA. It can be observed that the capacity of water absorption by the gel decreases significantly with increasing concentration of A6ACA from $30 \mathrm{wt} . \%$ to $70 \mathrm{wt} . \%$. This is because the networks formed with high concentration of A6ACA are strongly cross-linked and this fact reflects in a smaller expansion of matrix and, consequently, smaller volume of water diffuses into the interior of the matrix [19, 20]. Similar behavior was presented by Aouada et al. [20] and Pourjavadi et al. [21]. 

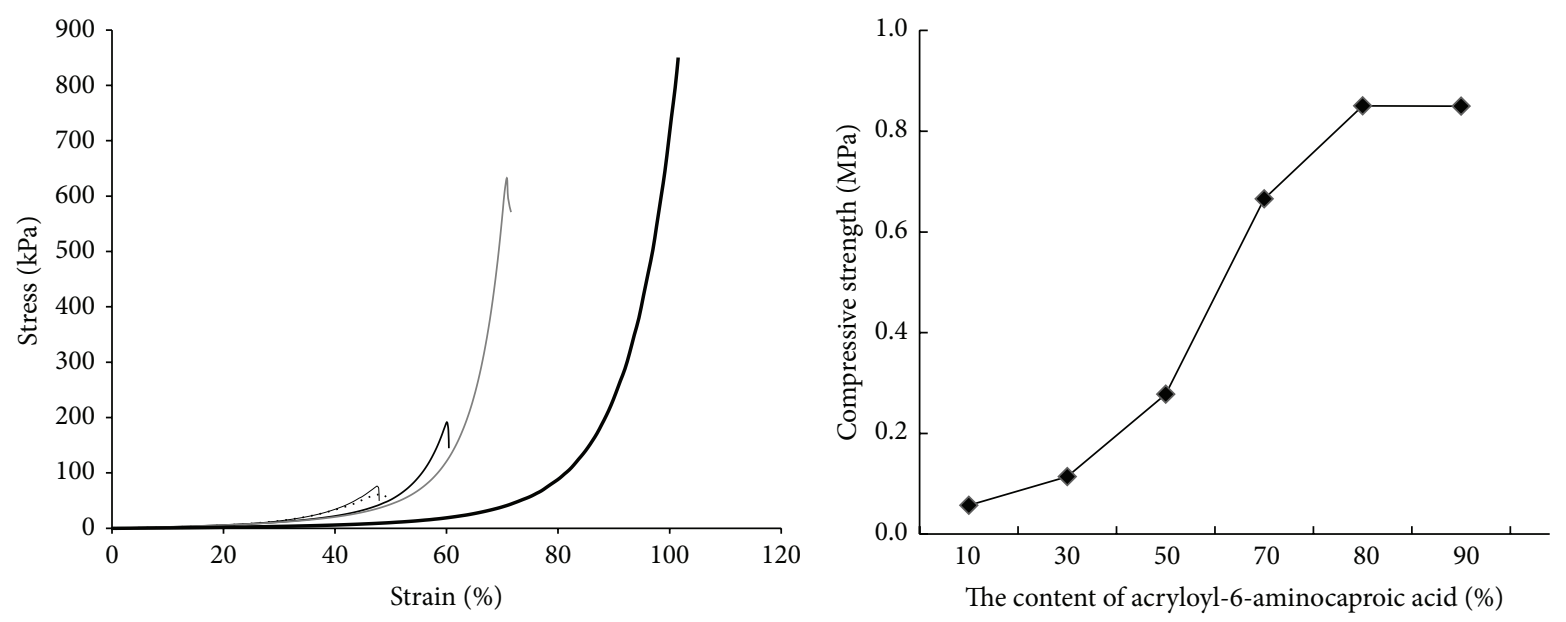

$\begin{array}{ll}\text { (a) } & \\ \text { (b) } & (\mathrm{d}) \\ \text { (c) } & (\mathrm{e})\end{array}$

(a)

(b)

Figure 4: Effects of A6ACA content on mechanical properties of hydrogels (A6ACA content: (a) 10 wt.\%; (b) 30 wt.\%; (c) 50 wt.\%; (d) 70 wt.\%; (e) 80 wt.\%)

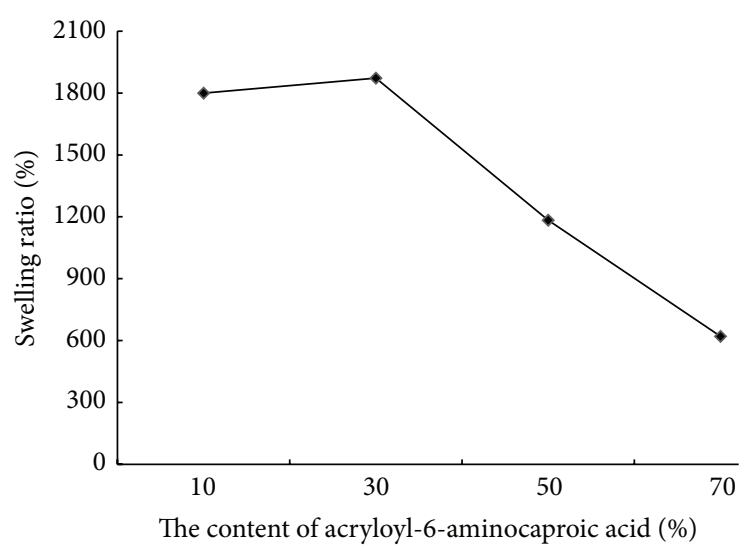

Figure 5: The effect of A6ACA on the swelling ratio of hydrogels.

3.4. The Self-Repairing Performance of Hydrogels. Hydrogels were synthesized using different concentrations of A6ACA, which presents an important role in the self-healing characteristics of the hydrogel. Hydrogels without A6ACA will lose self-repairing capacity. The increase of A6ACA concentration in the CMCS hydrogels will lead to increased mechanical properties (stress increased from $76 \mathrm{KPa}$ to $632 \mathrm{KPa}$ and strain increased from $47 \%$ to $71 \%$ (Figure 6)), which lead to both physical and chemical cross-links, and mechanical healing efficiencies (stress healing efficiencies increased from $19 \%$ to $48 \%$ and strain healing efficiencies increased from $66 \%$ to $85 \%$ (Figure 6 )), which lead to physical crosslinks $[15$, 22]. Even at the highest A6ACA concentrations of $70 \mathrm{wt} . \%$, the mechanical performance in healed hydrogels is lower than in single hydrogels because in the hydrogel the covalent cross-links and noncovalent cross-links both bear loads and hydrogen bond is the main reason for self-healing ability. Failure in single hydrogels involves breakage of both covalent bonds and intramolecular hydrogen bonds, whereas failure in healed hydrogels involves only breakage of intermolecular hydrogen bonds across the interface, which is a phenomenon that has been observed in other supramolecular self-healing polymer systems [22].

\section{Conclusions}

In summary, we have demonstrated that self-healing can be achieved in chemically cross-linked systems through introduction of pendant side chains, offered by A6ACA. Such materials will increase the reliability and service life of thermosetting polymers used in a wide variety of applications.

\section{Conflict of Interests}

The author declares that there is no conflict of interests regarding the publication of this paper. 

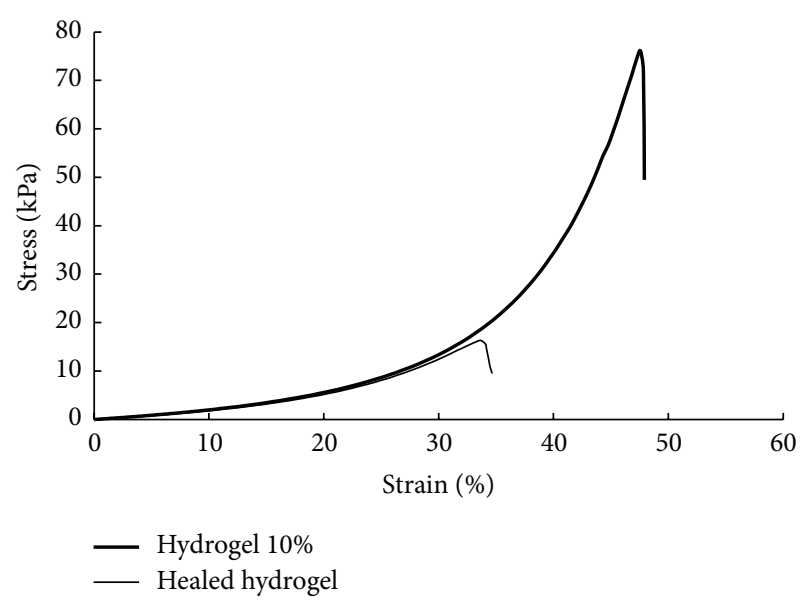

(a)

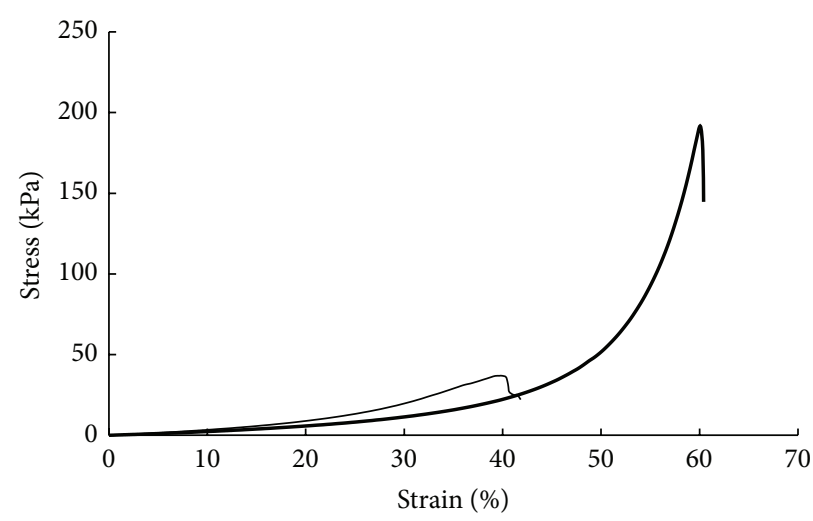

— Hydrogel 50\%
Healed hydrogel

(c)

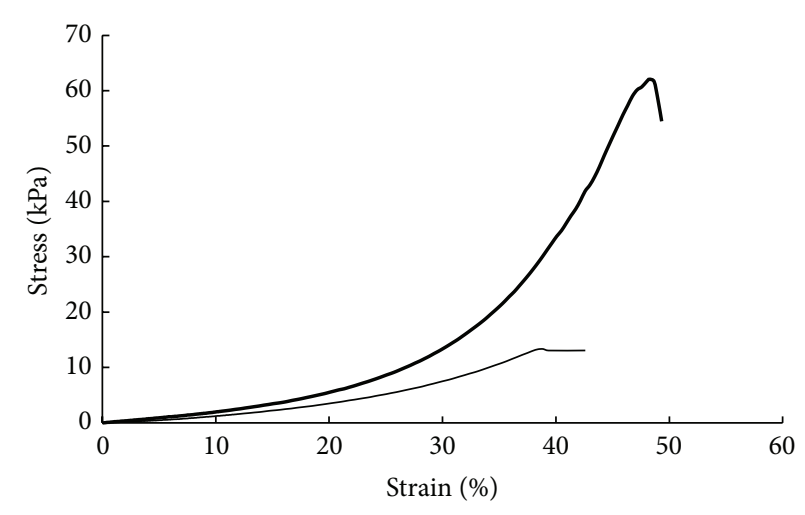

— Hydrogel 30\% — Healed hydrogel

(b)

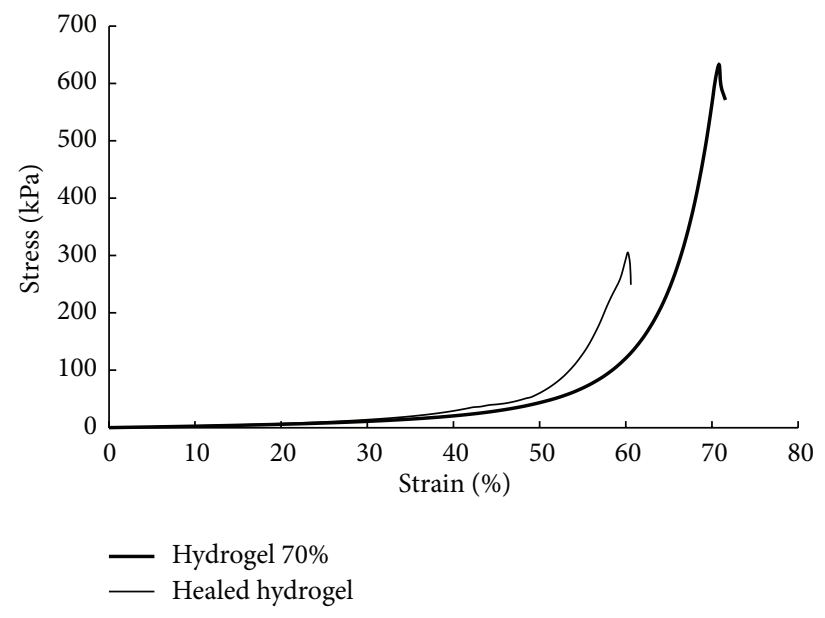

(d)

Figure 6: The self-healing properties of hydrogels (the content of A6ACA in the system was (a) 10 wt.\%; (b) 30 wt.\%; (c) 50 wt.\%; (d) 70 wt.\%).

\section{Acknowledgment}

This paper is supported by the "Fundamental Research Funds for the Central Universities.”

\section{References}

[1] E. Guibal, "Interactions of metal ions with chitosan-based sorbents: a review," Separation and Purification Technology, vol. 38, no. 1, pp. 43-74, 2004.

[2] K. Junka, O. Sundman, J. Salmi, M. Österberg, and J. Laine, "Multilayers of cellulose derivatives and chitosan on nanofibrillated cellulose," Carbohydrate Polymers, vol. 108, no. 1, pp. 3440, 2014.

[3] Y. Xie, H. Qiao, Z. Su, M. Chen, Q. Ping, and M. Sun, "PEGylated carboxymethyl chitosan/calcium phosphate hybrid anionic nanoparticles mediated hTERT siRNA delivery for anticancer therapy," Biomaterials, vol. 35, no. 27, pp. 7978-7991, 2014.

[4] N. Masaki, T. Yoshinori, Y. Hiroyasu, and H. Akira, "Redoxresponsive self-healing materials formed from host-guest polymers," Nature Communications, vol. 2, article 511, 6 pages, 2011.
[5] J. F. Duan, X. J. Zhang, J. X. Jiang et al., "The synthesis of a novel cellulose physical gel," Journal of Nanomaterials, vol. 2014, Article ID 312696, 6 pages, 2014.

[6] P. Cordier, F. Tournilhac, C. Soulié-Ziakovic, and L. Leibler, "Self-healing and thermoreversible rubber from supramolecular assembly," Nature, vol. 451, no. 7181, pp. 977-980, 2008.

[7] M. Burnworth, L. Tang, J. R. Kumpfer et al., "Optically healable supramolecular polymers," Nature, vol. 472, no. 7343, pp. 334337, 2011.

[8] Q. G. Wang, J. L. Mynar, M. Yoshida et al., "High-water-content mouldable hydrogels by mixing clay and a dendritic molecular binder," Nature, vol. 463, no. 7279, pp. 339-343, 2010.

[9] N. Holten-Andersen, M. J. Harrington, H. Birkedal et al., "pHinduced metal-ligand cross-links inspired by mussel yield selfhealing polymer networks with near-covalent elastic moduli," Proceedings of the National Academy of Sciences of the United States of America, vol. 108, no. 7, pp. 2651-2655, 2011.

[10] X. Chen, M. A. Dam, K. Ono et al., "A thermally re-mendable cross-linked polymeric material," Science, vol. 295, no. 5560, pp. 1698-1702, 2002.

[11] B. Ghosh and M. W. Urban, "Self-repairing oxetane-substituted chitosan polyurethane networks," Science, vol. 323, no. 5920, pp. 1458-1460, 2009. 
[12] G. V. Kolmakov, R. Revanur, R. Tangirala et al., "Using nanoparticle-filled microcapsules for site-specific healing of damaged substrates: creating a 'repair-and-go' system,' ACS Nano, vol. 4, no. 2, pp. 1115-1123, 2010.

[13] D. L. Han and L. F. Yan, "Supramolecular hydrogel of chitosan in the presence of graphene oxide nanosheets as 2D cross-linkers," ACS Sustainable Chemistry \& Engineering, vol. 2, no. 2, pp. 296300, 2014.

[14] M. Vatankhah-Varnoosfaderani, S. Hashmi, A. Ghavami Nejad, and F. J. Stadler, "Rapid self-healing and triple stimuli responsiveness of a supramolecular polymer gel based on boroncatechol interactions in a novel water-soluble mussel-inspired copolymer," Polymer Chemistry, vol. 5, no. 2, pp. 512-523, 2014.

[15] A. Phadke, C. Zhang, B. Arman et al., "Rapid self-healing hydrogels," Proceedings of the National Academy of Sciences of the United States of America, vol. 109, no. 12, pp. 4383-4388, 2012.

[16] B. P. Lee, P. B. Messersmith, J. N. Israelachvili, and J. H. Waite, "Mussel-inspired adhesives and coatings," Annual Review of Materials Research, vol. 41, pp. 99-132, 2011.

[17] H. Kono and M. Zakimi, "Preparation, water absorbency, and enzyme degradability of novel chitin- and cellulose/chitinbased superabsorbent hydrogels," Journal of Applied Polymer Science, vol. 128, no. 1, pp. 572-581, 2013.

[18] K. Kabiri, H. Omidian, M. J. Zohuriaan-Mehr, and S. Doroudiani, "Superabsorbent hydrogel composites and nanocomposites: a review," Polymer Composites, vol. 32, no. 2, pp. 277-289, 2011.

[19] P. J. Flory, "Phase equilibria in polymer systems," in Principles of Polymer Chemistry, chapter 13, Cornell University Press, Ithaca, NY, USA, 1953.

[20] F. A. Aouada, M. R. de Moura, A. F. Rubira et al., "Birefringent hydrogels based on PAAm and lyotropic liquid crystal: optical, morphological and hydrophilic characterization," European Polymer Journal, vol. 42, no. 10, pp. 2781-2790, 2006.

[21] A. Pourjavadi, S. H. Barzegar, and F. Zeidabadi, "Synthesis and properties of biodegradable hydrogels of $\kappa$-carrageenan grafted acrylic acid-co-2-acrylamido-2-methylpropanesulfonic acid as candidates for drug delivery systems," Reactive \& Functional Polymers, vol. 67, no. 7, pp. 644-654, 2007.

[22] S. R. White, N. R. Sottos, P. H. Geubelle et al., "Autonomic healing of polymer composites," Nature, vol. 409, no. 6822, pp. 794-797, 2001. 

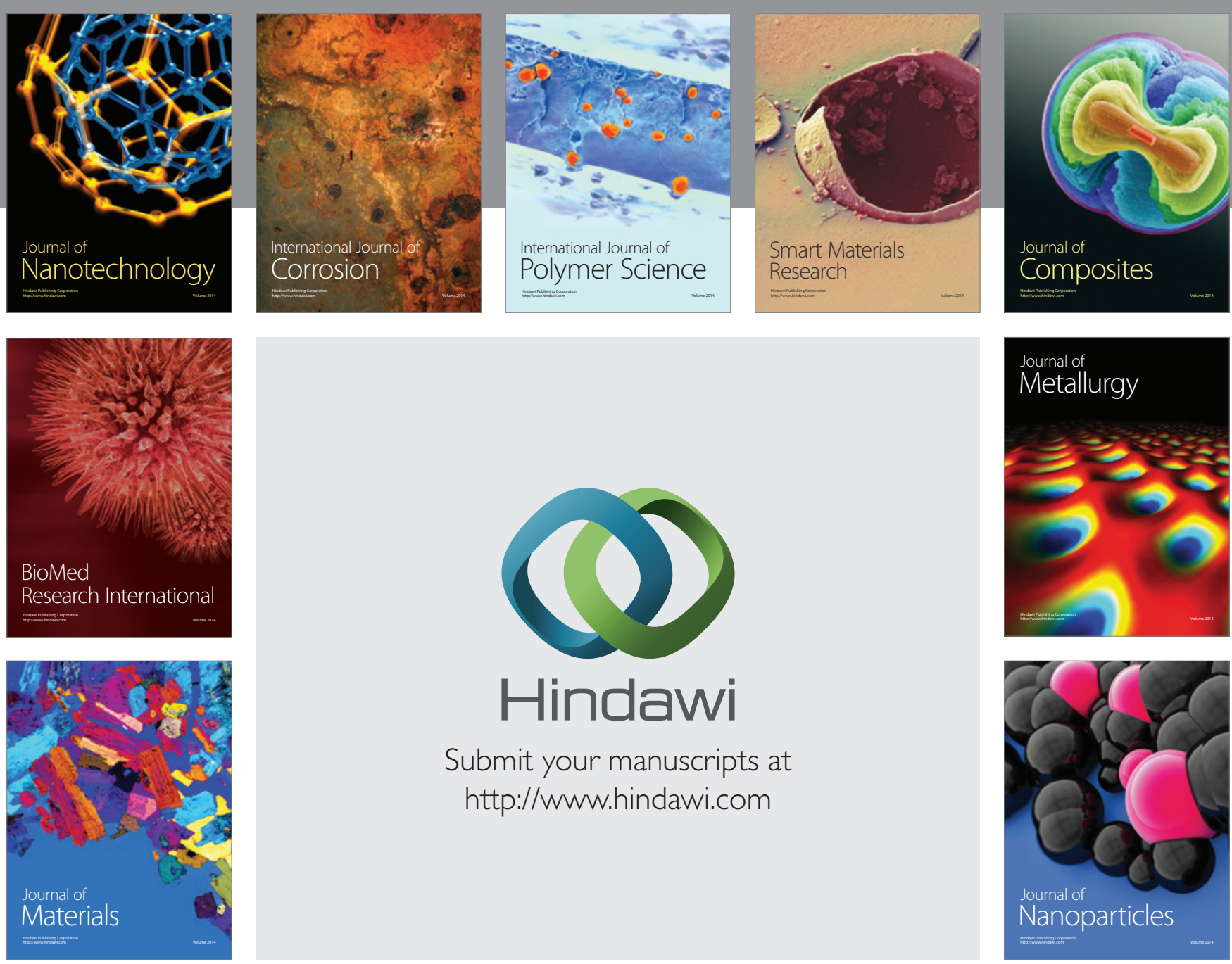

Submit your manuscripts at http://www.hindawi.com
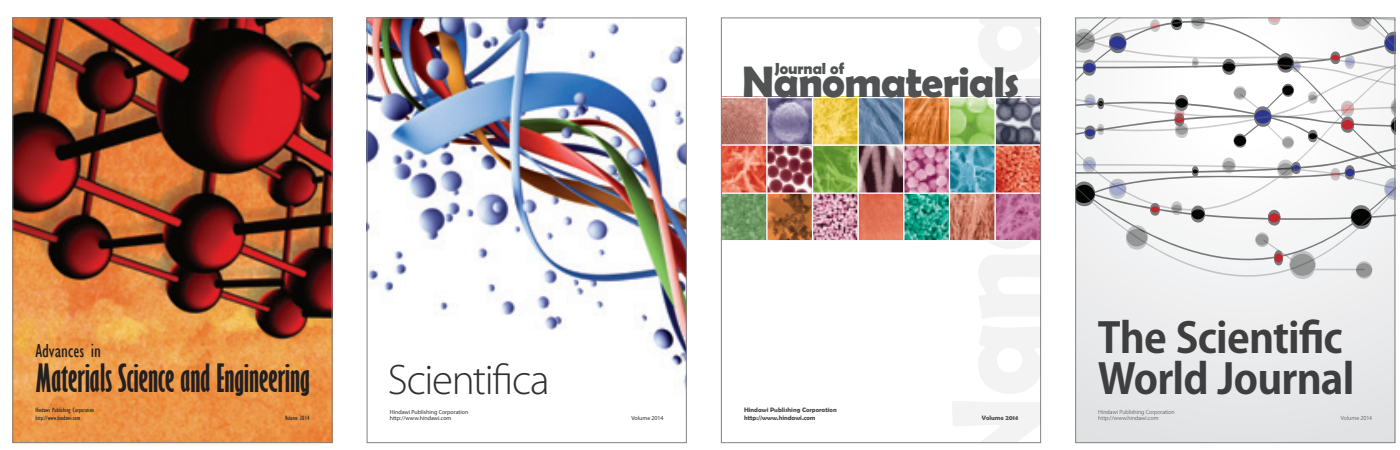

\section{The Scientific World Journal}
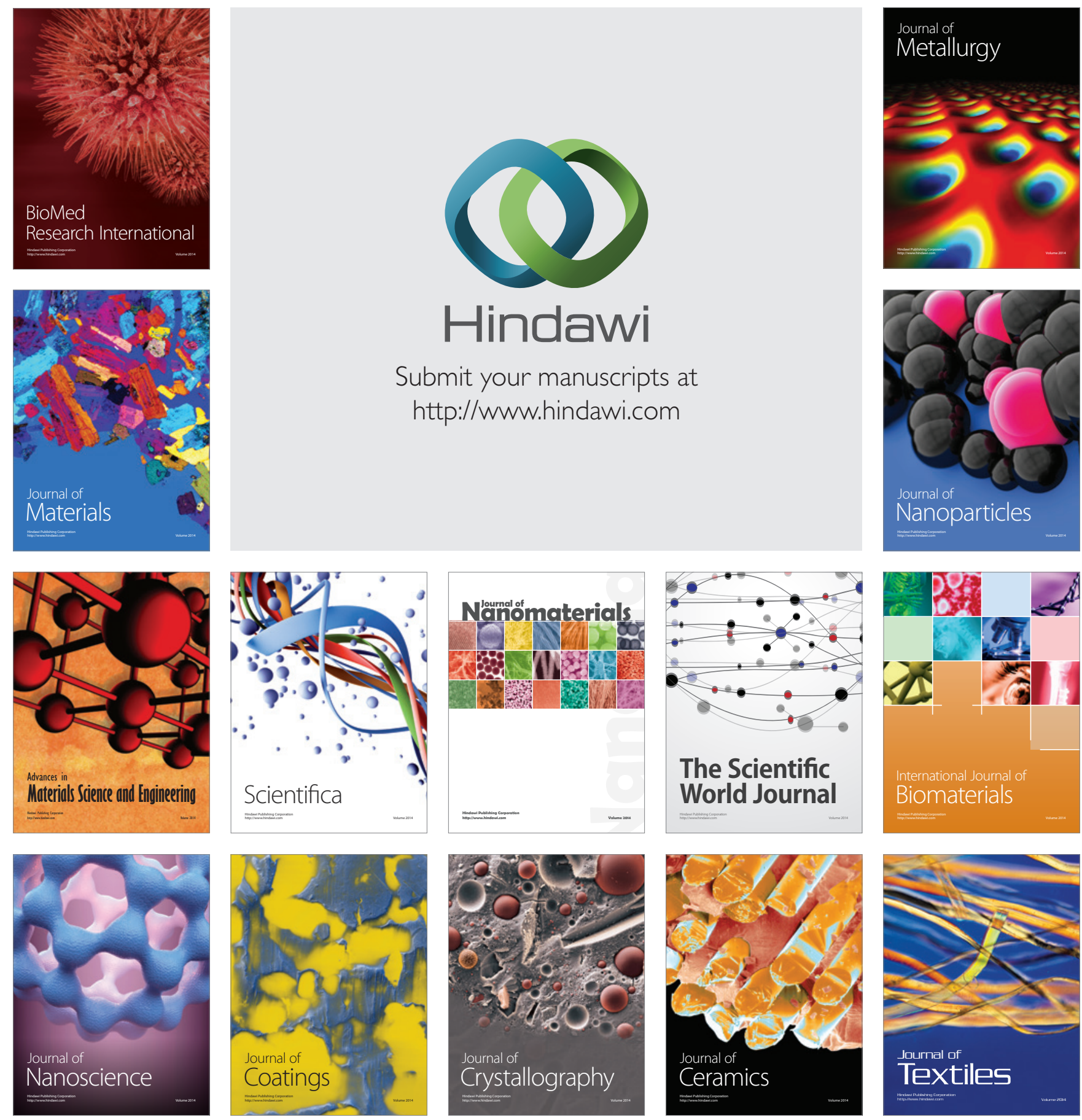